\title{
Prioritization of Receivers for Minimum Possible Error Boundary in Time Difference of Arrival Algorithm
}

\author{
Sourav Kaity ${ }^{1 *}$, Biswapati Jana ${ }^{2}$, P.K. Das Gupta ${ }^{3}$ and Lalatendu Das ${ }^{4}$ \\ ${ }^{1,4}$ Integrated Test Range, DRDO, Chandipur-756025 \\ ${ }^{2}$ Vidyasagar University, Midnapore-721102 \\ ${ }^{3}$ Proof and Experimental Establishment, DRD0, Chandipur-756025 \\ E-mail: *souravkaity@gmail.com
}

\begin{abstract}
A lot of passive target tracking techniques are available to find out the unknown target location. In this literature, Time difference of arrival (TDOA), a widely used passive target tracking technique, is used to derive the position of the target. By applying cross-correlation techniques on signals received by two different receivers one hyperbolic equation can be formed. With the help of a minimum four receiving stations, a unique intersecting point can be derived from hyperbolic equations which give the position of a target precisely. The accuracy of the target position depends upon the geometric location of the receivers with respect to the target location. A simulation study was carried out with seven numbers of receivers. We considered all thirty-five combinations taking four receivers at a time out of seven. From this simulation study, a unique relation between target position measurement errors with the average range difference error is established. With the help of the above relation, receivers can be prioritized and four receivers could be placed in best geographical locations. By considering four high prioritized receivers minimum target position measurement error could be achieved. An attempt was focused to draw the error boundary, error factor of target position measurement with the range of the target. And it is clear that the error factor is varying linearly with the range of the target.
\end{abstract}

Keywords: TDOA, Hyperbolic Equation, Range Difference, Error Boundary

\section{INTRODUCTION}

A never-ending demand for finding the location and improving the measurement accuracy of target location either static or dynamic has been a most discussed topic among scientists as well as some technology-oriented organization. This helps largely in military prospects where the opponent's incoming target is estimated within milliseconds. Apart from this, it helps in emergency evacuation situations, navigation purposes, tracking a person, various search operations, etc. The aviation sector has also significantly modernized with the help of positional location technology.

For measurement of target location, there exists several passive target localization techniques likes Time on arrival (TOA), Angle of Arrival (AOA), Time Difference of Arrival (TDOA), Received Signal Strength (RSS), etc. and also some hybrid techniques which are a combination of two different localization algorithm mentioned above (Deligiannis et al., 2010). AOA measures the angle of the source with respect to sensors. The power present in a received signal varies from a shorter distance to longer. RSS technique computes the position parameters by this energy level of the received signal. In TOA techniques the travel time of signal converted in distance to get positional parameters. Each method has its own advantages and disadvantages according to the application.

For an exact positional value, the TOA method requires strict clock synchronization between source and receiver station (Shin and Sung 2002). To avoid such a synchronization problem and to improve the accuracy of a target, TDOA techniques used. It is a cross-correlation technique and also known as hyperbolic position location techniques. 
A fundamental approach presented by Krizman et al. (1997) with respect to TDOA considering basic radio frequency position location strategies. To find source location from noisy instances through range differences have proposed by Friedlander (1987). A weighted matrix was derived for the least square estimator and a simulation formed to analyze the results. The observation was only valid if the range difference measurement is unbiased.

An approach presented by Kossonou et al. (2014) is a non-iterative method and has less complex computing based on Chan's method for TDOA. Position estimation is done in two-steps, in first related position parameters of TDOA are computed then applied with position algorithm in the second step. The positioning algorithm should be obtained in a perfect channel.

Asynchronous time difference of arrival positioning system is proposed by He et al. (2017) where the position was calculated without time synchronization with all the receiver anchor and target nodes.

A positional algorithm is developed for a twodimensional context, considering there are one transmitter and multiple receivers (Chan and Ho 1994). A set of equations formed from TDOA measurements is solved by the least square method. Here only TDOA estimation error was considered.

Due to the non-linearity of the hyperbolic equation, the TDOA measurement is uncertain. A comparing study between Monte Carlo based method and the gradient search algorithm was presented using a non-linear least square framework (Gustafsson 2003).

A detailed derived method was presented to find a position with four fixed stations (Potluri 2001). A derived model was formed from the hyperbolic equation and the simulation result shows the accuracy level of the experiment.

For greater accuracy, we extend this approach for our experiment to calculate TDOA and also analysis the error. In section 2 we described our approach. Simulation results and error analysis was done in section 3 and finally, in section 4, we concluded the paper with our end result.

\section{TDOA ALGORITHM FOR POSITION MEASUREMENT}

In this section, we solved the localization problem using time difference of arrival (TDOA) methods. There are four receivers present which give us a hyperbolic equation.
By solving these hyperbolic equations lead us to get the positional value. But it is very complex to solve those equations and hence the error analysis becomes a difficult task. This is due to the nonlinearity of the hyperbolic equation.

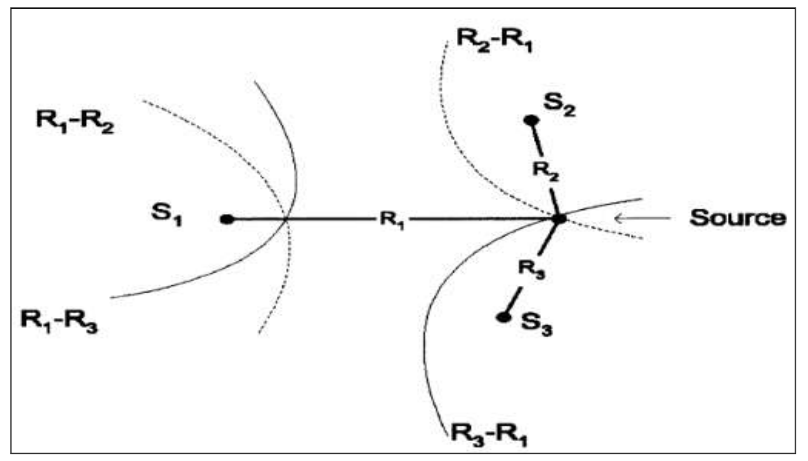

Fig. 1: A Sample Hyperbolic Position Location Solution (Three Receivers)

At first range difference between two receivers is calculated by the difference in time of arrival of the signal. The time of arrival (TOA) of two geo-spatial receivers was taken into consideration. The range can be calculated from the product of TDOA and the propagation speed. The propagation speed is equivalent to the speed of the light. This range difference yields to the hyperbolic equation between two receiver stations. In range difference measurement if the unknown quantity to be determined is equal to the number of the equation then there exists a unique solution. If there exist multiple intersections between two hyperbolae, then ambiguity exists in estimated position value.

Basically solving these hyperbolic equations is a challenge. The Taylor series expansion is used to linearize the equation. It is an iterative method. It can give an exact solution unless the initial guess is not correct. A method proposed by Frag (1990) gave a correct derivation to the linearization problem where the number of an unknown variable is equal to the number of the hyperbolic equation. But this method suffers because of inborn squaring operation. In terms of complexity computation, it is less intensive than the Taylor series expansion. Chan and Ho (1994) proposed execution method by solving repetitive estimation. This method is superior than the Taylor series and the Fang's method.

If $(\mathrm{x}, \mathrm{y}, \mathrm{z})$ is source transmitter location and $\left(x_{i}, y_{i}, z_{i}\right)$ is the location of the $\mathrm{i}^{\text {th }}$ receiver, is a range of source transmitter from the $\mathrm{i}^{\text {th }}$ receiver given by

$$
R_{i}=\sqrt{\left(x_{i}-x\right)^{2}+\left(y_{i}-y\right)^{2}+\left(z_{i}-z\right)^{2}}
$$




\section{Prioritization of Receivers for Minimum Possible Error Boundary}

Then the range differences of four different combinations of the source to the receiver are calculated and the equation for the same represented below

$$
\begin{aligned}
& R_{i j}=R_{i}-R_{j}=\sqrt{\left(x_{i}-x\right)^{2}+\left(y_{i}-y\right)^{2}+\left(z_{i}-z\right)^{2}}- \\
& \sqrt{\left(x_{j}-x\right)^{2}+\left(y_{j}-y\right)^{2}+\left(z_{j}-z\right)^{2}} \\
& R_{i k}=R_{i}-R_{k}=\sqrt{\left(x_{i}-x\right)^{2}+\left(y_{i}-y\right)^{2}+\left(z_{i}-z\right)^{2}}- \\
& \sqrt{\left(x_{k}-x\right)^{2}+\left(y_{k}-y\right)^{2}+\left(z_{k}-z\right)^{2}} \\
& R_{k j}=R_{k}-R_{j}=\sqrt{\left(x_{k}-x\right)^{2}+\left(y_{k}-y\right)^{2}+\left(z_{k}-z\right)^{2}}- \\
& \sqrt{\left(x_{j}-x\right)^{2}+\left(y_{j}-y\right)^{2}+\left(z_{j}-z\right)^{2}} \\
& R_{k l}=R_{k}-R_{l}=\sqrt{\left(x_{k}-x\right)^{2}+\left(y_{k}-y\right)^{2}+\left(z_{k}-z\right)^{2}-} \\
& \sqrt{\left(x_{l}-x\right)^{2}+\left(y_{l}-y\right)^{2}+\left(z_{l}-z\right)^{2}}
\end{aligned}
$$

Now The time difference of arrival between receiver $\mathrm{i}$ and receiver $\mathrm{j}$ can be expressed as

$$
t_{i j}=\frac{R_{i}-R_{j}}{c}
$$

Where $\mathrm{i}$ is greater than $\mathrm{j}$ and $\mathrm{c}$ is the velocity of light.

Let $x_{i}(t)$ and $x_{j}(t)$ be signals received at $\mathrm{i}^{\text {th }}$ and $\mathrm{j}^{\text {th }}$ receiver respectively. One way to calculate the time difference $t_{i j}$ of the signal received at $i^{\text {th }}$ and $j^{\text {th }}$ receiver is given by means of the standard cross-correlation function.

Using nonlinear regression, this equation can be converted to the form a hyperbola. Once enough hyperbolas have been calculated, the position of the transmitter can be calculated by finding the intersection.

Let, $\left(x_{0}, y_{0}, z_{0}\right)$ is the source location. Then range from ith receiver to source is $r_{i}$.

$$
r_{i}=\sqrt{\left(x_{0}-x_{i}\right)^{2}+\left(y_{0}-y_{i}\right)^{2}+\left(z_{0}-z_{i}\right)^{2}}
$$

We would require four equations to obtain a solution here, which can be obtained by expressions of $r_{i}, r_{j}, r_{k}, r_{l}$

$$
\begin{aligned}
& r_{i}-r_{j}=r_{i j} \\
& r_{i}-r_{k}=r_{i k} \\
& r_{k}-r_{j}=r_{k j} \\
& r_{k}-r_{l}=r_{k l}
\end{aligned}
$$

Where,

$$
\begin{aligned}
& r_{i j}=\sqrt{\left(x_{0}-x_{i}\right)^{2}+\left(y_{0}-y_{i}\right)^{2}+\left(z_{0}-z_{i}\right)^{2}}- \\
& \sqrt{\left(x_{0}-x_{j}\right)^{2}+\left(y_{0}-y_{j}\right)^{2}+\left(z_{0}-z_{j}\right)^{2}} \\
& r_{i k}=\sqrt{\left(x_{0}-x_{i}\right)^{2}+\left(y_{0}-y_{i}\right)^{2}+\left(z_{0}-z_{i}\right)^{2}}- \\
& \sqrt{\left(x_{0}-x_{k}\right)^{2}+\left(y_{0}-y_{k}\right)^{2}+\left(z_{0}-z_{k}\right)^{2}}
\end{aligned}
$$

$$
\begin{aligned}
& r_{k j}=\sqrt{\left(x_{0}-x_{k}\right)^{2}+\left(y_{0}-y_{k}\right)^{2}+\left(z_{0}-z_{k}\right)^{2}}- \\
& \sqrt{\left(x_{0}-x_{j}\right)^{2}+\left(y_{0}-y_{j}\right)^{2}+\left(z_{0}-z_{j}\right)^{2}} \\
& r_{k l}=\sqrt{\left(x_{0}-x_{k}\right)^{2}+\left(y_{0}-y_{k}\right)^{2}+\left(z_{0}-z_{k}\right)^{2}}- \\
& \sqrt{\left(x_{0}-x_{l}\right)^{2}+\left(y_{0}-y_{l}\right)^{2}+\left(z_{0}-z_{l}\right)^{2}}
\end{aligned}
$$

The four equations given above can be solved and rearranged to obtain two plane equations as follows

$$
\begin{aligned}
& y=A x_{0}+B z_{0}+C \\
& y=D x_{0}+E z_{0}+F
\end{aligned}
$$

Where,

$$
\begin{aligned}
& A=\frac{R_{i j} x_{j i}-R_{i j} x_{k i}}{R_{i j} y_{k i}-R_{i k} y_{j i}} \\
& B=\frac{R_{i k} z_{j i}-R_{i j} z_{k i}}{R_{i j} y_{k i}-R_{i k} y_{j i}}
\end{aligned}
$$

$C=\frac{R_{i k}\left[R_{i j}^{2}+x_{i}^{2}-x_{j}^{2}+y_{i}^{2}-y_{i}^{2}+z_{i}^{2}+z_{j}^{2}\right]-R_{i j}\left[R_{i k}^{2}+x_{i}^{2}-x_{k}^{2}+y_{i}^{2}-y_{k}^{2}+z_{i}^{2}+z_{k}^{2}\right]}{2\left[R_{i j} y_{k i}-R_{i k} y_{j i}\right]}$

$\begin{aligned} D & =\frac{R_{k l} x_{j k}-R_{k j} x_{l k}}{R_{k j} y_{l k}-R_{k l} y_{j k}} \\ E & =\frac{R_{k l} z_{j k}-R_{k j} z_{l k}}{R_{k j} y_{l k}-R_{k l} y_{j k}}\end{aligned}$

$E=\frac{R_{k l} z_{j k}-R_{k j} z_{l k}}{R_{k j} y_{l k}-R_{k l} y_{j k}}$

$F=\frac{R_{k l}\left[R_{k j}^{2}+x_{k}^{2}-x_{j}^{2}+y_{k}^{2}-y_{j}^{2}+z_{k}^{2}+z_{j}^{2}\right]-R_{k j}\left[R_{k l}^{2}+x_{k}^{2}-x_{l}^{2}+y_{k}^{2}-y_{l}^{2}+z_{k}^{2}+z_{l}^{2}\right]}{2\left[R_{k j} y_{l k}-R_{k l} y_{j k}\right]}$

Solving Equations (16) and (17), gives a linear equation for $\mathrm{x}_{0}$ in terms of $\mathrm{z}_{0}$.

$$
\begin{aligned}
& A x_{0}+B z_{0}+C=D x_{0}+E z_{0}+F \\
& x_{0}=G z_{0}+H
\end{aligned}
$$

Where,

$$
\begin{aligned}
& G=\frac{E-B}{A-D} \\
& H=\frac{F-C}{A-D}
\end{aligned}
$$

Substituting Equation (25) back into simplefied representation Equation (16) gives a linear equation for $\mathrm{y}_{0}$ in terms of $z_{0}$.

$$
y_{0}=I z_{0}+J
$$

Where,

$$
\begin{aligned}
& I=A G+B \\
& J=A H+C
\end{aligned}
$$

Equations (25) and (28), if substituted back into simplefied representation of Equation (13) give 
$K=R_{i k}^{2}+x_{i}^{2}-x_{k}^{2}+y_{i}^{2}-y_{k}^{2}+z_{i}^{2}-z_{k}^{2}+2 x_{k i} H+2 y_{k i} J$

And,

$L=2\left[x_{k i} G+y_{k i} I+2 z_{k i}\right]$

$M=4 R_{i k}^{2}\left[G^{2}+I^{2}+1\right]-L^{2}$

$N=8 R_{i k}^{2}\left[G\left(x_{i}-H\right)+I\left(y_{i}-J\right)+z_{i}\right]+2 L K$

$0=4 R_{i k}^{2}\left[\left(x_{i}-H\right)^{2}+\left(y_{i}-J\right)^{2}+z_{i}^{2}\right]-K^{2}$

Final solution is as follows

$$
\begin{aligned}
& z_{0}=\frac{N}{2 M}+\sqrt{\left(\frac{N}{2 M}\right)^{2}-\frac{O}{M}} \\
& y_{0}=I z_{0}+J \\
& x_{0}=G z_{0}+H
\end{aligned}
$$

Finally after solving, the position of the target is obtained.

\section{RESULT AND ANALYSIS}

A python script was written to test on sample data because it is a high-level language and analysis of data is smoother and easier as compared to other programming languages. Source position is considered as $x, y, z$. The ranges differences $R_{i j}, R_{i k}, R_{k j}$ and $R_{k l}$ were calculated from the derived method above. This time difference of arrival means range difference is taken as input and by solving those hyperbolic equation mentioned above we found the source position as . This method applied to the projectile path of 1800 samples. The maximum range of $100 \mathrm{~km}$ and height varies from zero to $35 \mathrm{~km}$ approximately. In this simulation total, seven different locations are considered for receiver deployment. The proposed algorithm for estimating position using the TDOA method takes input of four different receivers so simulation was run on a total of 35 different combinations. All combinations of receiver measurements in different planes were analyzed. The behavior of all kinds of measurement was analyzed throughout the total path followed by the target. For showing the results of simulation we have considered only the best case and worst case.

The below figure shows the relation between the Range and Height,

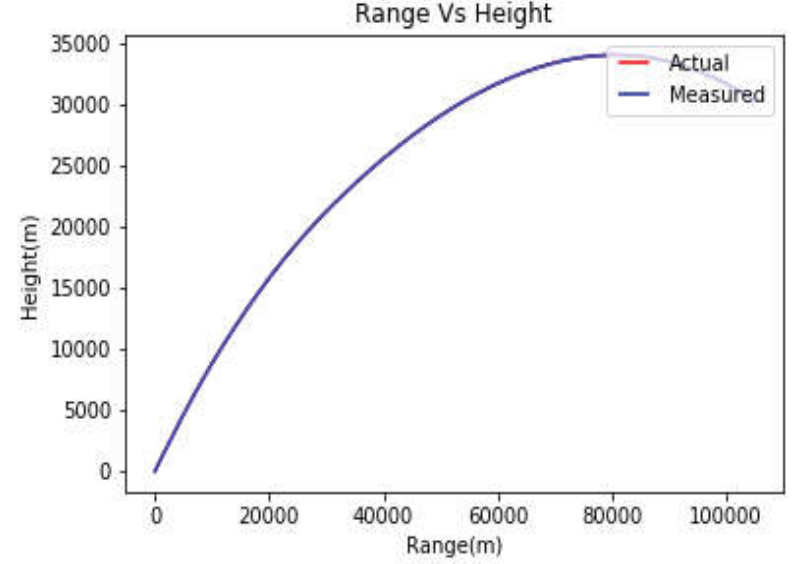

Fig. 2: Range vs Height (Best Case)

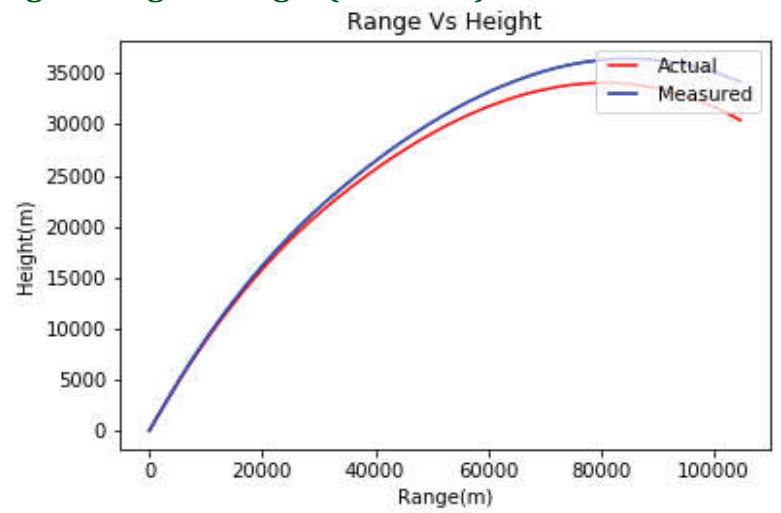

Fig. 3: Range vs Height (Worst Case)

Fig. 2 and Fig. 3 are showing the results of best and worst-case respectively in range vs height plane. In this case, it is clear that best-case results are overlapping but the differences of measurement are clearly visible in worstcase.

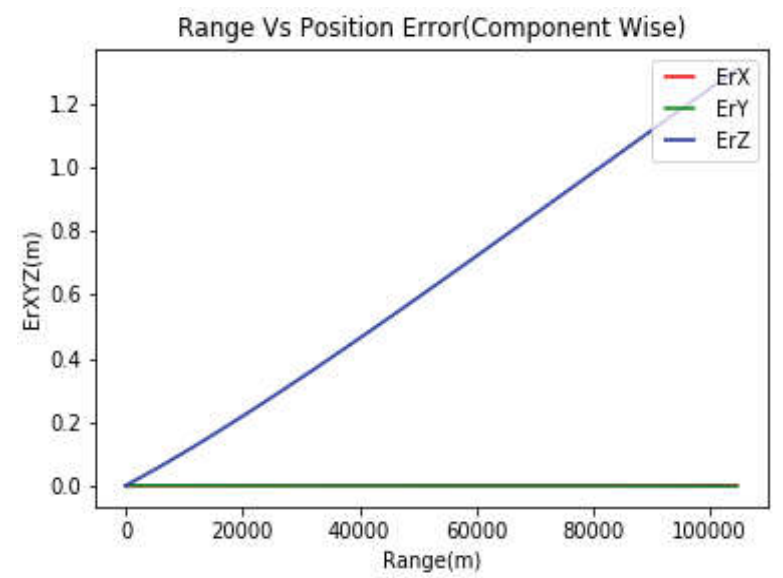

Fig. 4: Range vs Position Errors (Best Case) 


\section{Prioritization of Receivers for Minimum Possible Error Boundary}

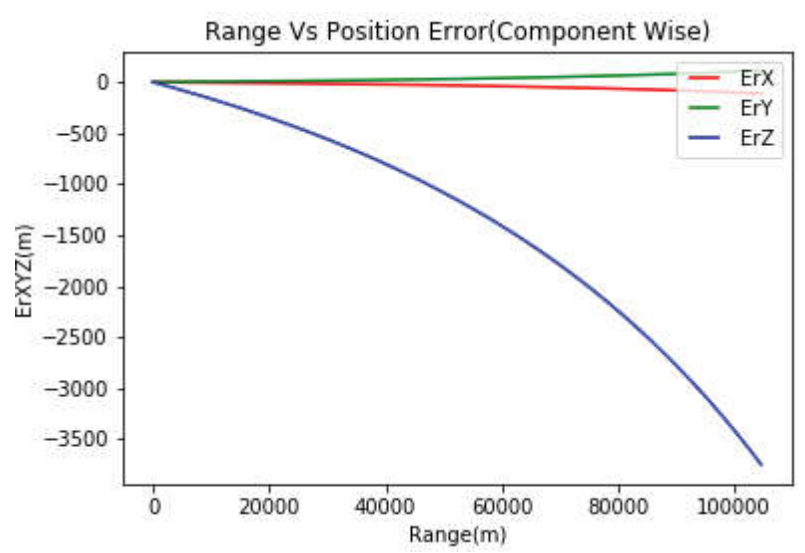

Fig. 5: Range vs Position Errors (Worst Case)

Fig. 4 and Fig. 5 are showing component-wise errors. An error was calculated by subtracting the actual and measured position in all three dimensions. Best case errors are in the order of 1 meter at $100 \mathrm{~km}$ range. The worstcase maximum error reached up to $3.5 \mathrm{~km}$. All component errors are increasing as the range of the target increases. A similar pattern of component error was observed in all cases. Another important observation is that all the cases the major contribution of error comes in the z-axis. As all the receivers are along the same $x-y$ plane so this hyperbolic equation solution produces more error in the z-axis.

Range difference error is calculated in the following manner.

$$
\begin{aligned}
& R_{i j} \text { Error }=R_{i j}-r_{i j} \\
& R_{i j} \text { Calculated from the time difference of arrival and } r_{i j}
\end{aligned}
$$
is calculated from the measured target position.

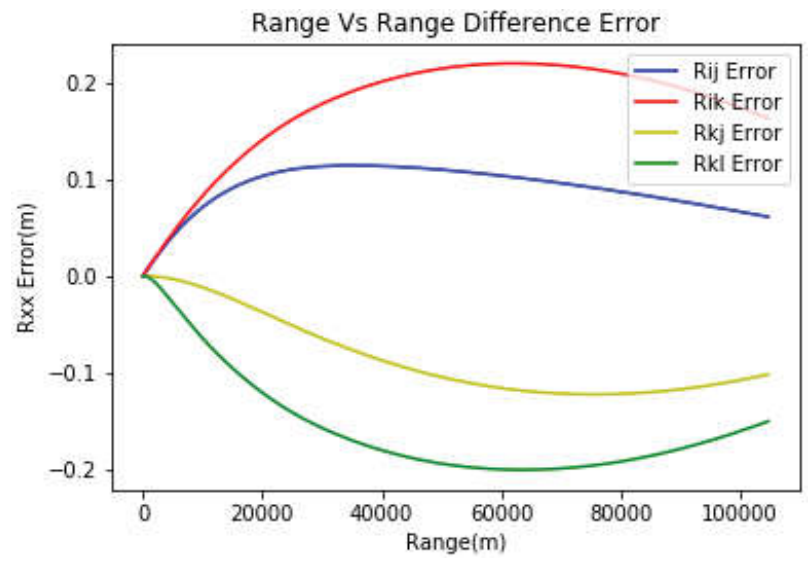

Fig. 6: Range vs Range Difference Errors (Best Case)

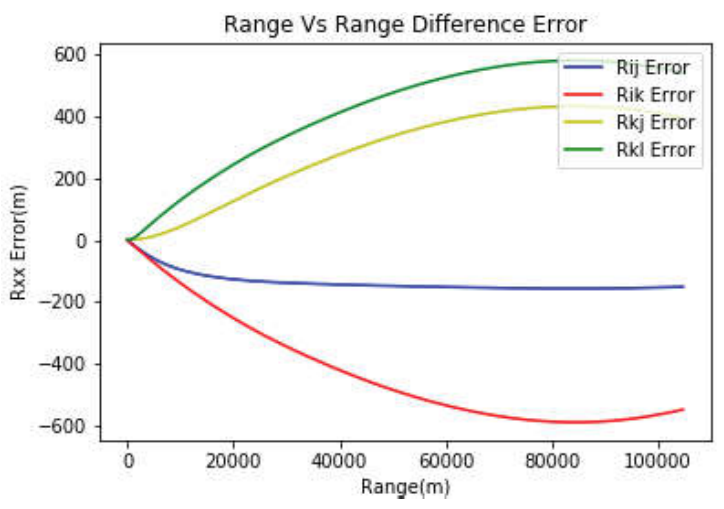

Fig. 7: Range vs Range Difference Errors (Worst Case)

Fig. 6 and Fig. 7 are showing the range difference error for all four combinations. Best case errors are in the order of .2 meter at $100 \mathrm{~km}$ range. The worst-case maximum error reached up to 600 meter. All combination errors are increasing as the range of the target increases. But at the end portion of the target location error is decreasing. As per the profile of the target, height is decreasing at the end portion. So the relation of range difference error depends upon range and height both. A similar pattern of error was observed in all cases.

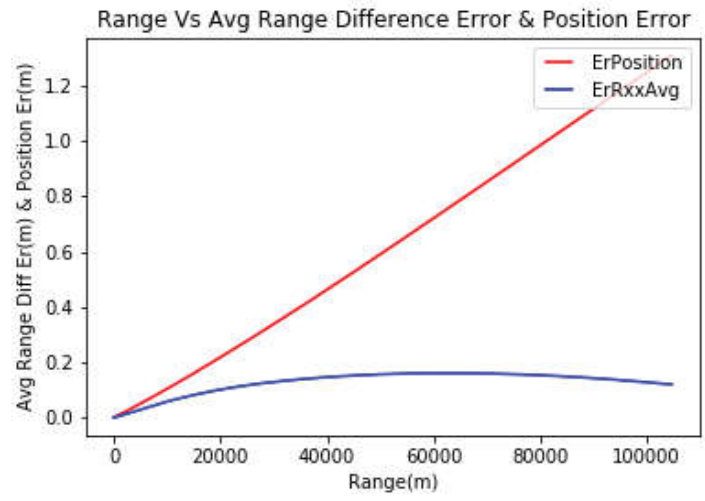

Fig. 8: Range vs Avg. Range Difference Errors and Position Error (Best Case)

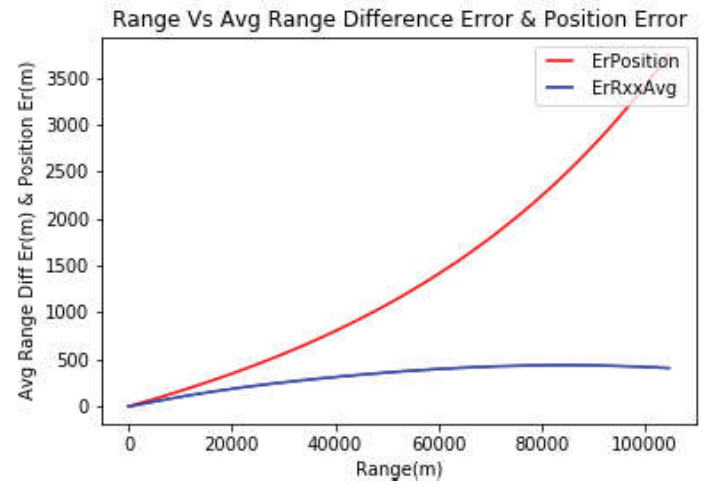

Fig. 9: Range vs Avg. Range Difference Errors and Position Error (Worst Case) 
Fig. 8 and Fig. 9 are showing the position error and average range difference error. An error was calculated by subtracting the actual and measured position. Best case errors are in the order of 1 meter at $100 \mathrm{~km}$ range. The worst-case maximum error reached up to $3.5 \mathrm{~km}$. Position error and average range difference error both are increasing as the range of the target increases. A similar pattern of error was observed in all cases.

To estimate the position measurement error average range difference error can be considered. In all cases, the relation between position errors varies with average range difference error linearly. And the same kind of results was observed in all cases from best case to worst case.

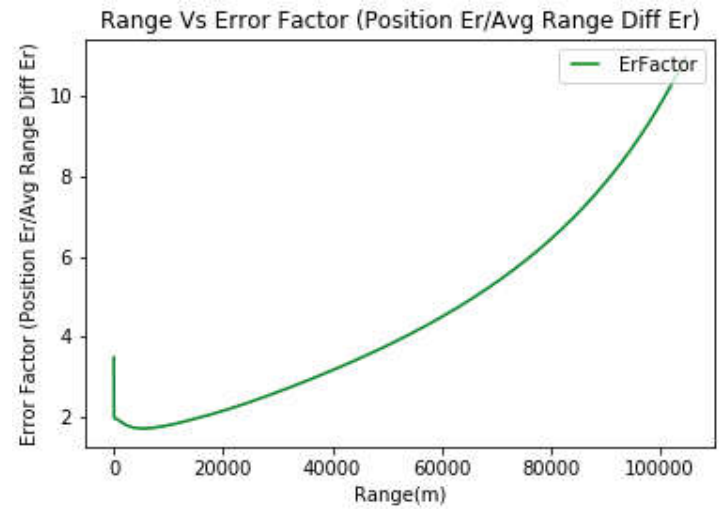

Fig. 10: Range vs Error Factor (Position Error/ Avg. Range Difference Errors) (Best Case)

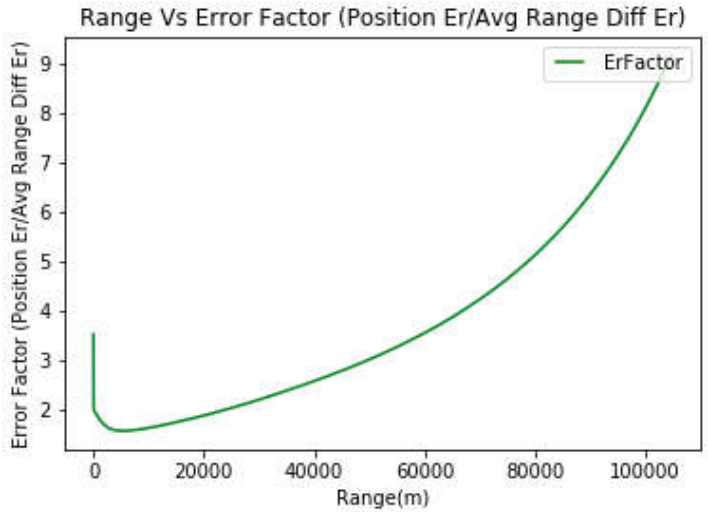

Fig. 11: Range vs Error Factor (Position Error/ Avg Range Difference Errors) (Worst Case)

An error factor is calculated just dividing position error by average range difference error.

Fig. 10 and Fig. 11 show the relation between error factors with range. The error factor is increasing as the range of target increases. A major observation is that error factor and range of target relationship is similar in all of the cases. In this best and worst case, it is clearly visible.

\section{CONCLUSIONS AND FUTURE WORK}

Target position finding algorithm using time difference of arrival considers four numbers of the receiver at a time to solve the hyperbolic equations. In our experiments, we considered four receivers at a time out of seven receivers, so we have a total of 35 numbers of combinations for the position finding of a target. As per the simulation results, it is clear that by calculating the average range difference error, we can prioritize the set of four-receiver location for minimum possible target position measurement error. The results were also able to establish the target position measurement error boundary. Error factor is calculated and the relation between target range and error factor is found linear irrespective of any combination of receivers. So our approach is able to find out the best possible combination of the receiver along with the target position measurement error boundary. The future study may be focused to find out more factors that can directly help in reducing the error boundary and prioritizing receivers. The probable target location zone could be formed using an error boundary. From this zone, one can simply assume the location of an incoming target. We are developing an automation tool, which will provide the position and measurement accuracy level of an incoming target with the help of the above result. It would be helpful for the aviation sector.

\section{REFERENCES}

Deligiannis N, Louvros S (2010) Hybrid TOA-AOA location positioning techniques in GSM networks. Wireless Personal Communications 54(2): 321-348.

Shin DH, Sung TK (2002) Comparisons of error characteristics between TOA and TDOA positioning. IEEE Transactions on Aerospace and Electronic Systems 38(1): 307-11.

Krizman KJ, Biedka TE, Rappaport TS. Wireless position location: fundamentals, implementation strategies, and sources of error. In 1997 IEEE 47th Vehicular Technology Conference. Technology in Motion; 1997 May 4; IEEE; 1997. pp. 919-923.

Friedlander B (1987) A passive localization algorithm and its accuracy analysis. IEEE Journal of Oceanic engineering 12(1): 234-245.

Kossonou KI, El Hillali Y, Bocquet M, Rivenq A, Assaad J (2014) NonIterative Three Dimensional Positioning Algorithm Based on Time Difference Of Arrival Technique. International Journal of Computer Science Issues; 2014 Mar 1; 11(2): 19.

He S, Dong X, Lu WS (2017) Localization algorithms for asynchronous time difference of arrival positioning systems. EURASIP Journal on Wireless Communications and Networking 1: 64

Chan YT, Ho KC (1994) A simple and efficient estimator for hyperbolic location. IEEE Transactions on signal processing 42(8): 1905-1915.

Gustafsson F, Gunnarsson F. Positioning using time-difference of arrival measurements. In IEEE International Conference on Acoustics, Speech, and Signal Processing; 2003 Apr 6 2003; IEEE. Proceedings 2003, pp. VI-553.

Potluri S (2001) Hyperbolic position location estimator with tdoas from four stations. MS thesis, Van Houten library, NJIT University.

Fang BT (1990) Simple solutions for hyperbolic and related position fixes. IEEE transactions on aerospace and electronic systems 26(5): 748-753. 